\title{
Current and future biomarkers in colorectal cancer
}

\author{
George Zarkavelis ${ }^{a, b}$, Stergios Boussiosa, ${ }^{a, b}$ Alexandra Papadaki ${ }^{a, b}$, Konstantinos H. Katsanosc, \\ Dimitrios K. Christodoulou' ${ }^{c}$, George Pentheroudakis ${ }^{\mathrm{a}, \mathrm{b}}$
}

Medical School, University of Ioannina; Society for Study of the Clonal Heterogeneity of Neoplasia, Ioannina, Greece

\section{Abstract}

\section{Introduction}

Colorectal cancer (CRC) is one of the leading causes of death among cancer patients worldwide. Risk factors associated with the incidence of CRC include older age, male sex, lifestyle, inflammatory bowel disease and a previous personal history of CRC. A positive family history is also strongly correlated with an increased relative risk of CRC diagnosis during lifetime. However, CRC is an indolent disease in its early stages and usually becomes symptomatic when it progresses to more advanced stages. Many efforts have been made to establish appropriate screening methods, but, to date, these remain

${ }^{a}$ Department of Medical Oncology, Medical School, University of Ioannina (George Zarkavelis, Stergios Boussios, Alexandra Papadaki, George Pentheroudakis); 'bociety for Study of the Clonal Heterogeneity of Neoplasia, Ioannina, Greece (George Zarkavelis, Stergios Boussios, Alexandra Papadaki, George Pentheroudakis); ${ }^{\mathrm{C}}$ Department of Gastroenterology, University Hospital of Ioannina, Faculty of Medicine, School of Health Sciences, University of Ioannina, Ioannina (Konstantinos H. Katsanos, Dimitrios K. Christodoulou), Greece

\section{Conflict of Interest: None}

Correspondence to: Dr Stergios Boussios MD, PhD, Medical Oncologist, Department of Medical Oncology, Medical School, University of Ioannina, Greece, Stavros Niarchou Avenue, 45110, Ioannina, Greece, e-mail: stergiosboussios@gmail.com

Received 10 July 2017; accepted 24 August 2017; published online 22 September 2017

DOI: https://doi.org/10.20524/aog.2017.0191 invasive, resulting in lower participation rates among the healthy population [1].

Recent advances in our knowledge of the molecular basis and cellular mechanisms of CRC have led to the adoption of specific molecular tests in every day clinical practice. Based on the test's results the patient's risk is stratified and therapy is determined. Molecular biomarkers that serve as prognostic factors are already in use and specific genomic mutations serving as predictive biomarkers are examined in formalinfixed tumor tissues. However, ongoing research for the identification of noninvasive biomarkers may lead to a new era in diagnosis, risk prediction and choice of treatment [2].

\section{Right- vs. left-sided CRC biology}

There is no uniform and consistent definition of the dividing point between right- and left-sided CRC. The most common distinction defines cancers proximal to the splenic flexure as right-sided and cancers at or distal to the splenic flexure as leftsided [3]. This cutoff point is often used because approximately two thirds of the transverse colon arises embryologically from the midgut, and only the distal one third arises from the hindgut (Table 1). Vascular supply has also been proposed as a defining characteristic of embryologic origin: the superior and inferior mesenteric arteries supply the midgut and hindgut, respectively.

There are also differences in gene expression profiles between normal right and left colon epithelium. Normal 
right colon has a higher expression of cytochrome P450 family genes than does the left colon [4]. Likewise, there are significant differences in the patterns of gene methylation between the right and left colon [5]. Notably, the prevalence of promoter methylation of the mismatch repair (MMR) gene hMLH1 and the O-6-methylguanine-DNA methyltransferase (MGMT) is significantly greater in normal right colon mucosa, especially in older women, suggesting epigenetic aberrations in preneoplastic right colon mucosa that may be reflected in subsequent right-sided adenocarcinoma biology [6].

There are different rates of mutation in key oncogenes and tumor suppressors between right- and left-sided CRC [7]. Mutations in BRAF V600E are significantly more common in right-sided CRC [8] (Table 1). Conversely, mutations in APC and TP53 are enriched in left-sided CRC [7]. Recently, different patterns of mutations in APC, TP53, and KRAS were identified as conferring differential prognoses in CRC [9]. Besides point mutations, potentially targetable amplifications of receptor tyrosine kinases, such as $E R B B 2$ and epidermal growth factor receptor (EGFR), are also more common in left-sided CRC [4].

The EGFR ligands epiregulin (EREG) and amphiregulin $(A R E G)$ are differentially expressed between right- and leftsided CRCs [10]. EREG and AREG expressions are significantly higher in left-sided CRC $[4,11]$, and are inversely correlated with promoter methylation [11].

There is differential prognosis by stage between patients with right- and left-sided CRC (Table 1). Retrospective studies suggest that right-sided tumors have a slightly better prognosis in stage II CRC, but a slightly worse prognosis in stage III disease, which is probably associated with the higher prevalence of good-prognosis microsatellite unstable (MSI-High) tumors in right-sided stage II cancers [3] (Table 1). Furthermore, analyses of prospective clinical trials of patients with stage III CRC who received adjuvant chemotherapy also demonstrated inferior progression-free survival (PFS) in those with rightsided CRC (hazard ratio [HR], 0.70; 95\% confidence interval [CI] 0.61-0.81) [12]. Patients who have metastatic CRC with a right-sided primary also have an inferior prognosis compared to those with a left-sided primary [13]. This was highlighted by a pooled analysis of 3 studies of 2027 evaluable patients treated with first-line chemotherapy, in which those with left-sided CRC had significantly better PFS and overall survival (OS) compared to those with right-sided CRC, even after adjustment for BRAF mutation and mucinous histology [14].

The primary CRC site is prognostic but not predictive of outcome with therapy based on the anti-angiogenic monoclonal antibody bevacizumab. An analysis of
2 prospective randomized controlled trials of chemotherapy, with or without bevacizumab, revealed that side is not a predictive biomarker for or against the benefit of bevacizumab. The statistical interaction test between side and bevacizumab use was nonsignificant [14].

However, the primary CRC site is both prognostic and predictive of benefit with anti-EGFR therapy among patients with KRAS wild-type, refractory, metastatic CRC. In the CO.17 randomized trial, among those with KRAS codon $12 / 13$ wildtype disease, there was a significant improvement in PFS with cetuximab in patients with a left-sided primary (HR 0.28 ; 95\%CI 0.18-0.45), whereas there was no difference in PFS among those with a right-sided primary (HR 0.73 ; 95\%CI 0.42-1.27; interaction $\mathrm{P}=0.002$ ) [15]. Additional retrospective studies also showed that patients with left-sided CRC had better PFS with anti-EGFR therapy compared with those with right-sided CRC, even among patients with KRAS/BRAF wildtype [4] and extended RAS and BRAF wild-type mutations [16] (Table 1).

\section{POLE and POLD1 germline mutations in familial CRC and polyposis}

Germline mutations in DNA polymerase 1 (POLE) and $\mathrm{d}(P O L D 1)$ have recently been identified in families with multiple colorectal adenomas and CRC. All reported cases carried POLE c.1270C >G (p.Leu424Val) or POLD1 c.1433G >A (p.Ser478Asn) mutations. Because of the scarcity of cases reported so far, an accurate clinical phenotype has not been defined. The two pathogenic mutations show dominant inheritance and confer high risk to multiple colorectal adenomas, large adenomas, early-onset CRC or multiple CRC. $P O L D 1$ p.S478N also confers an increased risk to endometrial cancer in female carriers [17-21].

Phenotypic data from the 69 carriers (29 families) of POLE/POLD1 exonuclease pathogenic mutations reported to date [17-21], point to an associated phenotype characterized by attenuated or oligo-adenomatous colorectal polyposis ( $>80 \%$ of $P O L E$ and $>60 \%$ of POLD1 mutation carriers were diagnosed with $\geq 2$ adenomas), CRC (60-64\% of carriers), and probably brain tumors (5.8\%). Gastroduodenal (mostly duodenal) adenomas were detected in $57.1 \%$ of carriers who underwent gastroduodenoscopy [20]. Moreover, the POLD1 phenotypic spectrum includes endometrial $(57.1 \%$ of female carriers) and breast (14.3\% of female carriers) tumors. All 21

Table 1 Molecular features of CRC by site

\begin{tabular}{|c|c|c|c|c|c|c|c|c|c|}
\hline & $\begin{array}{l}\text { CIMP } \\
\text { High }\end{array}$ & MSI High & $\begin{array}{l}\text { MLH1 } \\
\text { Methylation }\end{array}$ & $\begin{array}{l}B R A F \\
\text { mutation }\end{array}$ & $\mathrm{CIN}$ & CMS & Prognosis & $\begin{array}{l}\text { Outcome with } \\
\text { cetuximab }\end{array}$ & Embryogenesis \\
\hline Right-sided CRC & $\begin{array}{l}\text { High } \\
\text { prevalence }\end{array}$ & $\begin{array}{l}\text { High } \\
\text { prevalence }\end{array}$ & $\begin{array}{l}\text { High } \\
\text { prevalence }\end{array}$ & $\begin{array}{l}\text { High } \\
\text { prevalence }\end{array}$ & $\begin{array}{l}\text { Low } \\
\text { prevalence }\end{array}$ & 1,3 & Poorer & Inferior & Midgut \\
\hline Left-sided CRC & $\begin{array}{l}\text { Low } \\
\text { prevalence }\end{array}$ & $\begin{array}{l}\text { Low } \\
\text { prevalence }\end{array}$ & $\begin{array}{l}\text { Low } \\
\text { prevalence }\end{array}$ & $\begin{array}{l}\text { Low } \\
\text { prevalence }\end{array}$ & $\begin{array}{l}\text { High } \\
\text { prevalence }\end{array}$ & 2,4 & Better & Superior & Hindgut \\
\hline
\end{tabular}


POLE/POLD1 mutation carriers without cancer underwent resection of colorectal adenomas, indicating complete or very high expressivity of the associated phenotype. A better characterization of the syndrome is currently required to establish specific criteria for POLE and POLD1 exonuclease mutation screening and to help define the clinical management of mutation carriers.

\section{RAS mutational status}

KRAS proto-oncogene encodes a GTPase protein (KRAS) that has a substantial role in many molecular pathways. Approximately one third of CRC have point mutations in exon 2 (codons 12 and 13) or exon 3 of KRAS. Those mutations cause permanent activation of the RAS (RAS/RAF/MAPK) pathway. In addition, $15 \%$ of CRC carry mutations in exons 2, 3 and 4 of the NRAS gene [22]. These mutations can predict resistance to anti-EGFR therapy consisting of cetuximab or panitumumab monoclonal antibodies [23,24]. The clinical benefit of anti-EGFR antibody therapy is only observed in RAS wild-type tumors. Detection of KRAS mutations has become of high utility as a negative predictive factor, when deciding about the use of anti-EGFR therapy. In a retrospective series, the clinical characteristics of KRAS- and NRAS-mutated tumors were very similar, with the exceptions of an extremely low incidence of mucinous histology in NRAS-mutated tumors ( $4 \%$ vs. $26 \%, \mathrm{P}=0.012$ ) and a slightly lower prevalence of lung metastases ( $30 \%$ vs. $36 \%, \mathrm{P}=0.012)$ [25]. In terms of clinical outcome, worse median OS was observed in NRAS- and KRAS-mutated patients (25.6 months and 30.2 months, respectively) compared with all wild (42.7 months). Among 47 NRAS-mutated patients, 19 (40\%) received an anti-EGFR in advanced lines. Eight of them were evaluated for response to anti-EGFR therapy. Five, 2 and 1 patients received cetuximab plus irinotecan, cetuximab monotherapy and panitumumab monotherapy, respectively. At the first reassessment, 7 patients experienced disease progression and only 1 achieved initial disease stabilization, though it progressed after 8 weeks. Median PFS and OS were 2.4 and 8.5 months, respectively [25]. In patients with NRAS mutations, expanded RAS mutational status (both tumor KRAS and NRAS) should be evaluated in all candidates for anti-EGFR therapy [26]. There is a dire need for the identification and validation of positive predictive factors that are able to select, rather than exclude, tumors amenable to therapeutic EGFR axis modulation.

In another retrospective study, 84 patients with NRASmutant metastatic CRC were analyzed in terms of clinical characteristics, outcomes, and response to therapy [27]. OS was significantly shorter for NRAS exon 3 mutant metastatic CRC patients compared to RAS wild-type metastatic CRC patients (HR 2.85; 95\%CI 1.87-4.36, $\mathrm{P}<0.01$ ) and to NRAS exon 2 mutant metastatic CRC patients (HR 2.0; 95\%CI 1.04-4.0, $\mathrm{P}=0.039$ ). NRAS mutation represents a clinically and molecularly distinct subgroup of metastatic CRC, with increased left-sided colon primary. An increased proportion of RAS-mutant tumors, both KRAS and NRAS, was detected among African-American patients compared to Caucasians that was more pronounced for NRAS [27]. Approximately $68 \%$ of metastatic CRC in African Americans had a RAS mutation, possibly contributing to the poor outcomes among African Americans.

\section{BRAF mutations}

In the RAS signaling pathway ( $R A S / R A F / M A P K / R T K)$ the direct downstream target of KRAS is $B R A F$, which encodes serine threonine kinase proteins $[28,29]$. CRC that carry $B R A F$ mutations have been associated with poor prognosis. BRAF mutations are more frequent in right colon tumors, poorly differentiated, with a mucinous histology and infiltrating lymphocytes that are usually MSI-High [30]. Approximately $8 \%$ of CRC carry the distinct BRAF V600E mutation. This point mutation is mutually exclusive with KRAS mutations and is an adverse prognostic marker in advanced disease [31,32]. Contrary to the high response rates in melanoma, $B R A F$ V600E positive colorectal adenocarcinomas are resistant to $B R A F$ inhibition (i.e. vemurafenib), because of feedback activation of the EGFR/PI3K/AKT pathway $[33,34,35]$. Thus, resistance to $B R A F$ inhibitors in this subset of CRC cases has prompted the investigation of combined EGFR-BRAF-MEK inhibition, with or without chemotherapy [36] (Table 2). Minimal activity has recently been reported with the combination of $B R A F$ plus $M E K$ inhibitors in patients with $B R A F$ V600E-mutant metastatic colon cancer, in contrast to promising response rates of vertical $B R A F / M E K$ inhibition combined with anti-EGFR monoclonal antibodies in patients with refractory CRC [37].

In a retrospective study, Jones et al performed a detailed analysis of several large next-generation sequencing databases to determine the range of non-V600 mutations present in metastatic CRC [38]. They found that $2.2 \%$ of patients harbored a different $B R A F$ point mutation in their cancers, many of them within 10 bases of the 600 location. Obviously, in a common disease like metastatic CRC the absolute number of patients affected is significant. Clinical characteristics and outcomes for these patients were carefully collected, and the investigators demonstrated that patients with non-V600 $B R A F$-mutant metastatic CRC were significantly younger (58 vs. 68 years, respectively), more often male (65\% vs. $46 \%$, respectively), and less likely to have high-grade tumors (13\% vs. $64 \%$, respectively) or right-sided primary tumors $(36 \%$ vs. $81 \%$, respectively) compared to those with V600E BRAFmutant metastatic CRC. Additionally, patients with non-V600 BRAF mutations were more likely to have concomitant RAS mutations than patients with V600E BRAF mutations (26\% vs. $2 \% ; \mathrm{P}<0.001)$, but less likely to have MSI ( $6 \%$ vs. $30 \%$; $\mathrm{P}<0.001)$. Median OS was significantly longer in patients with non-V600 BRAF-mutant metastatic CRC as compared to those with either V600E $B R A F$-mutant or wild-type $B R A F$ metastatic CRC (60.7 vs. 11.4 vs. 43.0 months, respectively; $\mathrm{P}<0.001$ ). In multivariable analysis, non-V600 BRAF mutation was independently associated with improved OS (HR 0.18; 95\% CI $0.10-0.32 ; \mathrm{P}<0.001)$. It seems that non-V600 BRAF mutations 
Table 2 Activity of BRAF inhibitors and combinations of targeted therapies in BRAF V600E-mutated colorectal cancer

\begin{tabular}{|c|c|c|c|c|c|c|c|}
\hline Author/Reference & Year of publication & Treatment & Phase & $\begin{array}{c}\text { Number of } \\
\text { patients }\end{array}$ & OR (\%) & $\mathrm{SD}(\%)$ & PFS (months) \\
\hline Yaeger et al [36] & 2015 & Vemurafenib + panitumumab & $1 / 2$ & 15 & 13 & 53 & 3.2 \\
\hline Corcoran et al [37] & 2015 & Dabrafenib + trametinib & $1 / 2$ & 43 & 7 & 56 & 3.5 \\
\hline Gomez-Roca et al [84] & 2014 & Encorafenib & 1 & 18 & 0 & 67 & 4 \\
\hline Kopetz et al [85] & 2015 & Vemurafenib & 2 & 21 & 5 & 33 & 2.1 \\
\hline \multirow[t]{2}{*}{ Hyman et al [86] } & 2015 & Vemurafenib & 2 & 10 & 0 & 50 & 4.5 \\
\hline & & Vemurafenib + cetuximab & $1 / 2$ & 27 & 23 & 62 & 3.7 \\
\hline Hong et al [87] & 2016 & Vemurafenib + cetuximab + CPT11 & $1 b$ & 19 & 35 & - & 7.7 \\
\hline \multirow[t]{2}{*}{ Corcoran et al [88] } & 2016 & Dabrafenib + panitumumab & $1 / 2$ & 20 & 10 & 80 & 3.5 \\
\hline & & Dabrafenib + trametinib + panitumumab & $1 / 2$ & 91 & 21 & 59 & 4.2 \\
\hline \multirow[t]{2}{*}{ Tabernero et al [89] } & 2016 & Encorafenib + cetuximab + alpelisib & $1 \mathrm{~b} / 2$ & 52 & - & - & 5.4 \\
\hline & & Encorafenib + cetuximab & $1 b / 2$ & 50 & - & - & 4.2 \\
\hline
\end{tabular}

OR, objective response; $S D$, stable disease; $P F S$, progression-free survival

define a clinically distinct molecular subtype of metastatic CRC with an excellent prognosis, and that these findings have immediate clinical implications.

Several post hoc analysis of phase 3 randomized trials evaluated the predictive impact of the BRAF V600E mutation on the efficacy of anti-EGFR therapies. The results of these retrospective analyses did not reach statistical significance and were insufficiently powered to conclude whether or not the $B R A F$ V600E mutation is a biomarker of primary resistance to anti-EGFR agents in CRC [39-43]. Pietrantino et al conducted a meta-analysis of randomized trials to evaluate whether cetuximab or panitumumab in monotherapy or in combination with chemotherapy improved survival in patients with $B R A F$ V600E-mutated CRC [44]. In this meta-analysis, the addition of anti-EGFR agents to standard treatment in the RAS wildtype/BRAF V600E-mutated subgroup did not significantly improve PFS (HR 0.88; 95\%CI 0.67-1.14; $\mathrm{P}=0.33$ ) and OS (HR 0.91; 95\%CI 0.62-1.34; $\mathrm{P}=0.63$ ). However, another metaanalysis by Rowland et al evaluated whether the efficacy of anti-EGFR therapies differed according to the BRAF V600E mutational status by performing interaction tests [45]. Trials comparing anti-EGFR monoclonal antibodies to bevacizumab were excluded, taking into consideration that they were not comparable to those evaluating standard treatment with or without anti-EGFR agent. The reported HRs for PFS and OS which benefit from anti-EGFR therapies were 0.86 (95\%CI $0.61-1.21$ ) and 0.97 (95\%CI 0.67-1.41) for RAS wild-type/ $B R A F$ V600E-mutated tumors and 0.81 (95\%CI 0.70-0.95) and 0.62 (95\%CI $0.50-0.77$ ) for RAS wild-type/BRAF wild-type tumors. Tests of interaction of PFS and OS HRs between the two populations were not statistically significant $(\mathrm{P}=0.43$ and $\mathrm{P}=0.07$, respectively), suggested that the observed differences in survival benefit with anti-EGFR agents according to BRAF mutational status may be due to chance alone. Conversely, there may be insufficient evidence to justify the exclusion of anti-EGFR therapies for patients with RAS wild-type/BRAF V600E-mutated metastatic CRC.

\section{DNA MMR genes/MSI}

Microsatellites are repeating sequences of DNA in coding and non-coding areas, with a length of 1-6 base pairs; they are also known as simple sequence repeats [46]. MSI is the result of the MMR gene's inability to fix DNA errors occurring during replication. Somatic mutations in those repeating sequences, consisting mainly of insertions or deletions, lead to unstable genomic loci. The MMR genes are inactivated as a result of sporadic MLH1 promoter hypermethylation or germline mutations in MLH1, MSH2, MSH6 and PMS2 genes [47]. The germline genetic abnormality of MSI is the molecular basis of Lynch syndrome, also known as hereditary non-polyposis colon cancer (HNPCC) [48]. Localized CRC that are MMR-deficient (MSI-H) have a more favorable prognosis compared to MMR proficient (MSI-L) tumors. Stage II colon cancer patients who are MSI-H do not benefit from adjuvant 5-fluorouracil therapy and have a very good prognosis. Among patients with stage III tumors who participated in adjuvant chemotherapy trials, those whose tumors were MMR-deficient experienced better clinical outcomes compared to those with MMR proficient tumors [12]. However, the association of MMR-deficient status with prognosis is less robust in stage III than in stage II disease, and limited data are available in patients treated with the adjuvant FOLFOX (folinic acid, 5-fluorouracil, and oxaliplatin) regimen in contrast to fluorouracil alone. BRAF V600E mutations are associated with poorer outcomes in a metastatic setting [12,32] and are significantly enriched in sporadic colon cancers with MMR-deficient or MSI tumors [7,49]. In this regard, BRAF should be analyzed in conjunction with MMR for prognostic stratification of CRC.

The analysis of Sinicrope et al revealed a significant association of MMR-deficient CRC with better survival after recurrence, for cancers of the proximal rather than distal colon [12]. Likewise, analysis of KRAS mutations by primary tumor site suggested a significantly shorter survival after recurrence for patients with distal but not proximal cancers. 
Conversely, among patients with KRAS wild-type tumors, those with distal cancers achieved better survival than patients with proximal cancers. However, patients with stage III KRAS wild-type tumors, treated with FOLFOX with or without cetuximab, had similar survival, irrespective of the tumor site. In the metastatic setting, it seems that the treatment benefit of cetuximab is more pronounced for cancers of the distal colon than for proximal tumors [50]. Patients whose tumors harbored $B R A F$ V600E mutations had significantly poorer survival, and this was more prominent in distal tumors.

It is becoming increasingly common to test all newly diagnosed CRC cases for MSI, as it serves as a prognostic marker (stage II CRC), as a screening marker for Lynch syndrome, or as a future predictive biomarker (stage IV CRC). Le et al reported a phase II study in which patients with metastatic MMR-deficient (MSI-H) colon cancer were treated with the anti PDL1 antibody pembrolizumab, resulting in a $62 \%$ objective response rate compared to MSI-L tumors [51]. This was probably due to the high index of infiltrating lymphocytes and the increased expression of neoantigens in MSI-H tumors as a result of their high genomic instability [52].

\section{CpG island methylator phenotype (CIMP) - DNA hypermethylation status}

The main characteristic of CIMP+ phenotype is the high frequency of aberrantly methylated CpG islands (i.e. cytosine residues preceding guanines). It is more often observed in older patients, females and high-grade proximal tumors, which are usually BRAF-mutated and MMRdeficient (MSI-H) [49,53-56]. In addition, MLH1 promoter hypermethylation leading to MMR deficiency through gene silencing has been found in a subset of CIMP+ tumors [53]. CIMP status seems to be an emerging biomarker in CRC, because of its distinct mutations [57]. Furthermore, a plethora of hypermethylated genes, such as SLC5A8, ITGA4, SFRP2, CDKN2A, HLTF, and MGMT, seem to play crucial role in colon carcinogenesis [58]. Specific families of GTP-binding proteins, the septins, also participate in cell division. From the 13 known septins (SEPT1-SEPT13), SEPT9 gene silencing due to hypermethylation leads to a compromise of cell cycle functions and promotes carcinogenesis, making it a plausible future biomarker [59].

\section{EGFR/HER family}

EGFR is targetable in KRAS wild-type colorectal tumors by using the anti-EGFR antibodies cetuximab and panitumumab. However, after a few months of antibody administration, resistance to therapy emerges. HER2 has recently been evaluated as a possible resistance pathway to anti-EGFR antibody therapy in KRAS wild-type tumors [60]. In 5\% of KRAS non-mutated cancers, HER 2 is amplified and the administration of combined trastuzumab and lapatinib has been tested in a phase II study.
The dual HER2 blockade resulted in a $35 \%$ overall response rate and a median time to progression of about 5.5 months in heavily pretreated patients harboring HER2-amplified CRC. This is a hopeful indication that anti-HER2 therapy may be effective in this subset of metastatic CRC and HER2 expression may serve as a predictive biomarker [61].

EREG and AREG, which are EGFR ligands, have recently been investigated as possible biomarkers in the therapy of KRAS wild-type colorectal tumors receiving anti-EGFR antibody therapy. Studies of the mRNA expression of EREG and $A R E G$ have found controversial results regarding the response to anti-EGFR therapy while evaluating their role as predictive biomarkers [10,62,63]. A recent meta-analysis of studies investigating the impact of EREG and AREG mRNA levels in primary colon tumors came to the conclusion that they both serve as independent favorable prognostic and predictive biomarkers. High EREG and AREG expression was associated with longer PFS in patients receiving antiEGFR therapy, indicating the significance of EREG and AREG expression as biomarkers in CRC. More recently, evaluation of EREG and AREG levels and their association with PFS and OS was conducted in the FIRE1 clinical trial. Statistical analysis came to the conclusion that high EREG mRNA levels may serve as a positive prognostic marker regarding both PFS and OS, whereas high AREG levels did not affect patients' outcome $[64,65]$.

\section{Chromosomal instability}

Changes in the structure or number of chromosomes results in tumor karyotype alterations. Mutations that take place in oncogenes or tumor suppressor genes, along with defective telomeres, are the main causes of instability. Loss of heterozygosity, aneuploidy or amplifications are found in about $65 \%$ of CRC. Aneuploidy, which is an alteration in chromosomal number, is the result of defective mitotic checkpoint leading to abnormal segregation. Shorter telomeres, resulting from excessive telomere breakage, cause chromosomal instability, thus leading to carcinogenesis through transition from adenoma to carcinoma. Loss of heterozygosity, meaning loss of one parental allele, has been linked to CRC. Specifically, loss of heterozygosity in chromosome 18 has been linked to a poorer prognosis among CRC patients, especially those at stage II or III, who show lower survival rates compared to those who retain parental alleles in chromosome 18. Chromosomal instability and MMR status are the main pathways that give rise to CRC [66-68].

\section{TP53-APC/ $\beta$-catenin}

TP53 protein, encoded by the TP53 tumor suppressor gene located on the short arm of chromosome 17, has a regulatory role in cell growth arrest, DNA repair and apoptosis but also in oxidative stress, DNA damage and cell aberrant proliferation, 
thus maintaining cell cycle homeostasis. TP53 mutations result in dysfunctional TP53 protein, which has a critical role in tumor carcinogenesis. Mutated TP53 can be spotted in both malignant cells and in adenomas, and is expressed in about $60 \%$ of CRC. Point mutation in codon 72 - resulting in the substitution of proline to arginine - leads to dysfunction of the cell cycle "gatekeeper" that promotes the malignant process [69-70].

Germline APC mutations are the hallmark of familial adenomatous polyposis. Somatic mutations occurring in the $A P C$ gene activate the Wnt pathway in the early stages of colon carcinogenesis. The APC tumor-suppressor protein, encoded by the APC gene, has a crucial role in cellular processes while interacting and inactivating glycogen synthase-kinase-3 $\beta$ and $\beta$-catenin. Apart from somatic mutations that occur in the APC gene, promoter hypermethylation has also been recognized as a distinct cause of $A P C$ silencing [71-72].

\section{MicroRNAs}

Small RNAs, called microRNAs, play a key role in tumor suppression or growth. MicroRNAs are highly stable structures with a hairpin-loop shape and small size. They can be extracted not only from fixed tissues, but also from body fluids, especially peripheral blood. They are found in exosomes, distinct microvesicles secreted by tumor cells, achieving a high level of stability and avoiding degradation of the genomic material $[69,73]$. These RNA sequences can lead to silencing of targeted genes and interfere with the invasion and progression of tumors by epithelial mesenchymal transition to metastatic sites [74]. In CRC where $B R A F$ mutations are present, the expression of $m i R-31$ is noted, which could potentially be used as a diagnostic biomarker [75]. Furthermore, in KRAS wild-type tumors that respond to anti-EGFR antibody therapy, $m i R-99 a$ and $m i R-125 b$ may have a predictive role, whereas the expression of miR-181a correlates with a poor prognosis in this subset of patients [76]. Finally, poor responders to radiation therapy in rectal cancer cases have been found to express $m i R-622$ making it a plausible predictive marker [77]. Overall, expression of 500 different microRNAs has been noted in CRC.

\section{Blood and stool biomarkers}

Circulating tumor cells (CTC) originating from either the primary or metastatic sites, are detected in the sera of colon cancer patients. Their detection is associated with active disease, tumor progression and metastatic potential, making them a strong prognostic biomarker, but also providing the advantage of avoiding a new biopsy [78]. Following the isolation of CTC, genomic analyses can provide information regarding the biology of the tumor and its evolution, even when the patient is on therapy, achieving realtime monitoring of the disease and therapy effectiveness. However, given the small number of circulating tumor cells, circulating free DNA may constitute a more practical noninvasive biomarker [79].
Cell-free DNA (cfDNA) refers to DNA fragments originating from tumor cells that are detected in patients' sera or plasma. It can be extracted from peripheral blood and then examined for mutations and genomic abnormalities, providing real-time information about tumor progression [79]. cfDNA is more accurate than CTC regarding tumor burden and can be used as both a diagnostic and prognostic biomarker. It also has predictive potential in the assessment of antineoplastic therapy through molecular analysis and mutation identification. TP53 and KRAS mutations, MSI or loss of heterozygosity, along with DNA hypermethylation can be detected using cfDNA. cfDNA assays constitute a powerful tool for study of the molecular heterogeneity as well as the clonal divergence of a malignancy. Not surprisingly, the ability of circulating mRNA to resist degradation, mainly due to its presence in exosomes, has prompted investigation regarding the isolation of cellfree mRNA and the consequent use of reverse transcription polymerase chain reaction techniques in order to isolate a more accurate tumor signature using peripheral blood [80-81].

\section{Stool-based tests}

To date, fecal immunohistochemical tests and fecal occult blood tests are of utility for the detection of CRC, but show little specificity and sensitivity. Thus, detection of mutated DNA in stool may be a promising technique for the detection of CRC. The technical restriction of stool DNA detection is the fact that only $0.01 \%$ of total stool DNA derives from the patient, whereas the remainder comes from intestinal bacteria and microbial flora [82]. Specific panels that are able to detect mutated human DNA in stool could help in the diagnosis of CRC and contribute to the avoidance of invasive techniques for the diagnosis and molecular profiling of colon cancers [2,82].

\section{Molecular classification of CRC}

With the advent of molecular profiling and gene expression signatures, four distinct consensus molecular subtypes of CRC have been proposed. Current TNM staging remains the cornerstone for subsequent therapeutic decisions; however, there is a plethora of new molecular-based information for a CRC patient, indicating the implementation of personalized medicine according to the tumor's genetic signature. CMS1 MSI-immune CRCs are hypermutated, CIMP (+), frequently $B R A F$-mutated with MMR deficiency, and may respond to immunotherapy because of higher levels of tumor-infiltrating lymphocytes and tumor neoantigen load. These tumors have a dismal prognosis at their recurrence or in the metastatic setting. CMS2 Wnt-canonical tumors are characterized by Wnt and MYC activation with high somatic copy number alterations. CMS3-metabolic tumors have a high prevalence of KRAS mutations, discrete metabolic dysregulation, low CIMP and mixed MSI status. Finally, CMS4-mesenchymal tumors are the ones with high somatic copy number alterations, intense 
stromal infiltration and active angiogenesis, having the worse relapse-free and OS [83]. The molecular classification of CRC further reinforces the need for the establishment and use of molecular biomarkers.

\section{Concluding remarks}

CRC development is highly heterogeneous, with distinct molecular alterations taking place throughout the natural course of the disease. In everyday clinical practice, KRAS and NRAS mutations serve as predictive biomarkers for the selection of patients eligible for anti-EGFR therapy, with a benefit recorded only in RAS wild-type tumors. Mutations in BRAF have an adverse prognostic value and are associated with worse patient outcomes; they may also have a negative predictive value for the benefit from anti-EGFR therapy. According to the latest CRC guidelines, every newly diagnosed patient with stage II CRC must have the tumor checked for MMR status: in those who are MMR-deficient adjuvant 5-fluorouracil therapy is of no benefit, while the patient's prognosis is better. As our knowledge regarding the molecular landscape of colorectal carcinogenesis advances, new molecular biomarkers with prognostic and predictive information are being discovered. Liquid biopsies are a promising tool for real-time evaluation of the tumor clonal evolution, response to therapy, presence of minimal residual disease and acquired resistance. Such noninvasive biomarkers may lead to real-time tumor molecular classification and personalized treatment of CRC patients.

\section{References}

1. Kolligs FT. Diagnostics and Epidemiology of Colorectal Cancer. Visc Med 2016;32:158-164.

2. Coppedè F, Lopomo A, Spisni R, Migliore L. Genetic and epigenetic biomarkers for diagnosis, prognosis and treatment of colorectal cancer. World J Gastroenterol 2014;20:943-956.

3. Weiss JM, Pfau PR, O'Connor ES, et al. Mortality by stage for right- versus left-sided colon cancer: analysis of surveillance, epidemiology, and end results-Medicare data. J Clin Oncol 2011;29:4401-4409.

4. Missiaglia E, Jacobs B, D’Ario G, et al. Distal and proximal colon cancers differ in terms of molecular, pathological, and clinical features. Ann Oncol 2014;25:1995-2001.

5. Kaz AM, Wong CJ, Dzieciatkowski S, Luo Y, Schoen RE, Grady WM. Patterns of DNA methylation in the normal colon vary by anatomical location, gender, and age. Epigenetics 2014;9:492-502.

6. Menigatti M, Truninger K, Gebbers JO, Marbet U, Marra G, Schär P. Normal colorectal mucosa exhibits sex- and segmentspecific susceptibility to DNA methylation at the hMLH1 and MGMT promoters. Oncogene 2009;28:899-909.

7. Cancer Genome Atlas Network. Comprehensive molecular characterization of human colon and rectal cancer. Nature 2012;487:330-337.

8. Gonsalves WI, Mahoney MR, Sargent DJ, et al; Alliance for Clinical Trials in Oncology. Patient and tumor characteristics and BRAF and KRAS mutations in colon cancer, NCCTG/Alliance N0147. J Natl Cancer Inst 2014;106. doi: 10.1093/jnci/dju106.
9. Schell MJ, Yang M, Teer JK, et al. A multigene mutation classification of 468 colorectal cancers reveals a prognostic role for APC. Nat Commun 2016;7:11743.

10. Seligmann JF, Elliott F, Richman SD, et al. Combined epiregulin and amphiregulin expression levels as a predictive biomarker for panitumumab therapy benefit or lack of benefit in patients with RAS wild-type advanced colorectal cancer. JAMA Oncol 2016;2:633-642.

11. Lee MS, McGuffey EJ, Morris JS, et al. Association of CpG island methylator phenotype and EREG/AREG methylation and expression in colorectal cancer. Br J Cancer 2016;114:1352-1361.

12. Sinicrope FA, Shi Q, Allegra CJ, et al. Association of DNA mismatch repair and mutations in BRAF and KRAS with survival after recurrence in stage III colon cancers: a secondary analysis of 2 randomized clinical trials. JAMA Oncol 2017;3:472-480.

13. Schrag D, Weng S, Brooks G, Meyerhardt JA, Venook AP. The relationship between primary tumor sidedness and prognosis in colorectal cancer [abstract]. J Clin Oncol 2016;34:Abstract 3505.

14. Loupakis F, Yang D, Yau L. Primary tumor location as a prognostic factor in metastatic colorectal cancer. J Natl Cancer Inst 2015;107.

15. Brulé SY, Jonker DJ, Karapetis CS, et al. Location of colon cancer (right-sided versus left-sided) as a prognostic factor and a predictor of benefit from cetuximab in NCIC CO.17. Eur J Cancer 2015;51:1405-1414.

16. Moretto R, Cremolini C, Rossini D, et al. Location of primary tumor and benefit from anti-epidermal growth factor receptor monoclonal antibodies in patients with RAS and BRAF wild-type metastatic colorectal cancer. Oncologist 2016;21:988-994.

17. Palles C, Cazier JB, Howarth KM, et al; WGS500 Consortium. Germline mutations affecting the proofreading domains of POLE and POLD1 predispose to colorectal adenomas and carcinomas. Nat Genet 2013;45:136-144.

18. Valle L, Hernández-Illán E, Bellido F, et al. New insights into POLE and POLD1 germline mutations in familial colorectal cancer and polyposis. Hum Mol Genet 2014;23:3506-3512.

19. Elsayed FA, Kets CM, Ruano D, et al. Germline variants in POLE are associated with early onset mismatch repair deficient colorectal cancer. Eur J Hum Genet 2015;23:1080-1084.

20. Spier I, Holzapfel S, Altmüller J, et al. Frequency and phenotypic spectrum of germline mutations in POLE and seven other polymerase genes in 266 patients with colorectal adenomas and carcinomas. Int J Cancer 2015;137:320-331.

21. Bellido F, Pineda M, Aiza G, et al. POLE and POLD1 mutations in 529 kindred with familial colorectal cancer and/or polyposis: review of reported cases and recommendations for genetic testing and surveillance. Genet Med 2016;18:325-332.

22. Chang YY, Lin PC, Lin HH, et al. Mutation spectra of RAS gene family in colorectal cancer. Am J Surg 2016;212:537-544.

23. Cunningham D, Humblet $Y$, Siena S, et al. Cetuximab monotherapy and cetuximab plus irinotecan in irinotecan-refractory metastatic colorectal cancer. N Engl J Med 2004;351:337-345.

24. De Roock W, Claes B, Bernasconi D, et al. Effects of KRAS, BRAF, NRAS, and PIK3CA mutations on the efficacy of cetuximab plus chemotherapy in chemotherapy-refractory metastatic colorectal cancer: a retrospective consortium analysis. Lancet Oncol 2010;11:753-762.

25. Schirripa M, Cremolini C, Loupakis F, et al. Role of NRAS mutations as prognostic and predictive markers in metastatic colorectal cancer. Int J Cancer 2015; 136:83-90.

26. Sforza V, Martinelli E, Ciardiello F, et al. Mechanisms of resistance to anti-epidermal growth factor receptor inhibitors in metastatic colorectal cancer. World J Gastroenterol 2016;22:6345-6361.

27. Cercek A, Braghiroli MI, Chou JF, et al. Clinical features and outcomes of patients with colorectal cancers harboring NRAS mutations. Clin Cancer Res 2017;23:4753-4760. 
28. Ioannou M, Papamichali R, Samara M, et al. Diagnostic value of immunohistochemistry for the detection of the BRAF V600E mutation in colorectal carcinoma. J BUON 2016;21:618-625.

29. Fransén K, Klintenäs M, Osterström A, Dimberg J, Monstein HJ, Söderkvist P. Mutation analysis of the BRAF, ARAF and RAF-1 genes in human colorectal adenocarcinomas. Carcinogenesis 2004;25:527-533.

30. Pikoulis E, Margonis GA, Andreatos N, et al. Prognostic role of BRAF mutations in colorectal cancer liver metastases. Anticancer Res 2016;36:4805-4811.

31. Rajagopalan H, Bardelli A, Lengauer C, Kinzler KW, Vogelstein B, Velculescu VE. Tumorigenesis: RAF/RAS oncogenes and mismatch-repair status. Nature 2002;418:934.

32. Ogino S, Shima K, Meyerhardt JA, et al. Predictive and prognostic roles of BRAF mutation in stage III colon cancer: results from intergroup trial CALGB 89803. Clin Cancer Res 2012;18:890-900.

33. McArthur GA, Chapman PB, Robert C, et al. Safety and efficacy of vemurafenib in $\mathrm{BRAF}(\mathrm{V} 600 \mathrm{E})$ and $\mathrm{BRAF}(\mathrm{V} 600 \mathrm{~K})$ mutationpositive melanoma (BRIM-3): extended follow-up of a phase 3, randomised, open-label study. Lancet Oncol 2014;15:323-332.

34. Kopetz S, Chang GJ, Overman MJ, et al. Improved survival in metastatic colorectal cancer is associated with adoption of hepatic resection and improved chemotherapy. J Clin Oncol 2009;27:3677-3683.

35. Lito P, Pratilas CA, Joseph EW, et al. Relief of profound feedback inhibition of mitogenic signaling by RAF inhibitors attenuates their activity in BRAFV600E melanomas. Cancer Cell 2012;22:668-682.

36. Yaeger R, Cercek A, O'Reilly EM, et al. Pilot trial of combined BRAF and EGFR inhibition in BRAF-mutant metastatic colorectal cancer patients. Clin Cancer Res 2015;21:1313-1320.

37. Corcoran RB, Atreya CE, Falchook GS, et al. Combined BRAF and MEK inhibition with dabrafenib and trametinib in BRAF V600mutant colorectal cancer. J Clin Oncol 2015;33:4023-4031.

38. Jones JC, Renfro LA, Al-Shamsi HO, et al. (Non-V600) BRAF mutations define a clinically distinct molecular subtype of metastatic colorectal cancer. J Clin Oncol 2017;35:2624-2630.

39. Bokemeyer C, Van Cutsem E, Rougier P, et al. Addition of cetuximab to chemotherapy as first-line treatment for KRAS wild-type metastatic colorectal cancer: pooled analysis of the CRYSTAL and OPUS randomised clinical trials. Eur J Cancer 2012;48:1466-1475.

40. Douillard JY, Oliner KS, Siena S, et al. Panitumumab-FOLFOX4 treatment and RAS mutations in colorectal cancer. $N$ Engl J Med 2013;369:1023-1034.

41. Seymour MT, Brown SR, Middleton G, et al. Panitumumab and irinotecan versus irinotecan alone for patients with KRAS wild-type, fluorouracil-resistant advanced colorectal cancer (PICCOLO): a prospectively stratified randomised trial. Lancet Oncol 2013;14:749-759.

42. Karapetis CS, Jonker D, Daneshmand M, et al; NCIC Clinical Trials Group and the Australasian Gastro-Intestinal Trials Group. PIK3CA, BRAF, and PTEN status and benefit from cetuximab in the treatment of advanced colorectal cancer-results from NCIC CTG/AGITG CO.17. Clin Cancer Res 2014;20:744-753.

43. Peeters M, Price TJ, Cervantes A, et al. Final results from a randomized phase 3 study of FOLFIRI $\{+/-\}$ panitumumab for second-line treatment of metastatic colorectal cancer. Ann Oncol 2014;25:107-116.

44. Pietrantonio F, Petrelli F, Coinu A, et al. Predictive role of BRAF mutations in patients with advanced colorectal cancer receiving cetuximab and panitumumab: a meta-analysis. Eur J Cancer 2015;51:587-594.

45. Rowland A, Dias MM, Wiese MD, et al. Meta-analysis of BRAF mutation as a predictive biomarker of benefit from anti-EGFR monoclonal antibody therapy for RAS wild-type metastatic colorectal cancer. Br J Cancer 2015;112:1888-1894.

46. Zhang L. Immunohistochemistry versus microsatellite instability testing for screening colorectal cancer patients at risk for hereditary nonpolyposis colorectal cancer syndrome. Part II. The utility of microsatellite instability testing. J Mol Diagn 2008;10:301-307.

47. Ward R, Meagher A, Tomlinson I, et al. Microsatellite instability and the clinicopathological features of sporadic colorectal cancer. Gut 2001;48:821-829.

48. Tiwari AK, Roy HK, Lynch HT. Lynch syndrome in the $21^{\text {st }}$ century: clinical perspectives. QJM 2016;109:151-158.

49. Weisenberger DJ, Siegmund KD, Campan M, et al. CpG island methylator phenotype underlies sporadic microsatellite instability and is tightly associated with BRAF mutation in colorectal cancer. Nat Genet 2006;38:787-793.

50. Alan P, Venook DN. Impact of primary $\left(1^{\circ}\right)$ tumor location on overall survival (OS) and progression-free survival (PFS) in patients (pts) with metastatic colorectal cancer (mCRC): Analysis of CALGB/SWOG 80405 (Alliance). J Clin Oncol 2016;34:abstract $455 \mathrm{O}$.

51. Le DT, Uram JN, Wang H, et al. PD-1 blockade in tumors with mismatch-repair deficiency. N Engl J Med 2015;372:2509-2520.

52. Llosa NJ, Cruise M, Tam A, et al. The vigorous immune microenvironment of microsatellite instable colon cancer is balanced by multiple counter-inhibitory checkpoints. Cancer Discov 2015;5:43-51.

53. Grady WM, Carethers JM. Genomic and epigenetic instability in colorectal cancer pathogenesis. Gastroenterology 2008; 135:1079-1099.

54. Toyota M, Ahuja N, Ohe-Toyota M, Herman JG, Baylin SB, Issa JP. CpG island methylator phenotype in colorectal cancer. Proc Natl Acad Sci U S A 1999;96:8681-8686.

55. Hawkins N, Norrie M, Cheong K, et al. CpG island methylation in sporadic colorectal cancers and its relationship to microsatellite instability. Gastroenterology 2002;122:1376-1387.

56. Ogino S, Kawasaki T, Kirkner GJ, Kraft P, Loda M, Fuchs CS. Evaluation of markers for $\mathrm{CpG}$ island methylator phenotype (CIMP) in colorectal cancer by a large population-based sample. J Mol Diagn 2007;9:305-314.

57. Cohen SA, Wu C, Yu M, et al. Evaluation of CpG island methylator phenotype as a biomarker in colorectal cancer treated with adjuvant oxaliplatin. Clin Colorectal Cancer 2016;15:164-169.

58. Chen WD, Han ZJ, Skoletsky J, et al. Detection in fecal DNA of colon cancer-specific methylation of the nonexpressed vimentin gene. J Natl Cancer Inst 2005;97:1124-1132.

59. Kim MS, Froese CD, Estey MP, Trimble WS. SEPT9 occupies the terminal positions in septin octamers and mediates polymerizationdependent functions in abscission. J Cell Biol 2011;195:815-826.

60. Martin V, Landi L, Molinari F, et al. HER2 gene copy number status may influence clinical efficacy to anti-EGFR monoclonal antibodies in metastatic colorectal cancer patients. $\mathrm{Br} J$ Cancer 2013;108:668-675.

61. Siena S, Sartore-Bianchi A, Lonardi S, et al. Trastuzumab and lapatinib in HER2-amplified metastatic colorectal cancer patients (mCRC): The HERACLES trial. J Clin Oncol 2015;33:abstract 3508.

62. Razis E, Pentheroudakis G, Rigakos G, et al. EGFR gene gain and PTEN protein expression are favorable prognostic factors in patients with KRAS wild-type metastatic colorectal cancer treated with cetuximab. J Cancer Res Clin Oncol 2014;140:737-748.

63. Sebio A, Páez D, Salazar J, et al. Intergenic polymorphisms in the amphiregulin gene region as biomarkers in metastatic colorectal cancer patients treated with anti-EGFR plus irinotecan. Pharmacogenomics J 2014;14:256-262.

64. Stahler A, Heinemann V, Giessen-Jung C, et al. Influence of mRNA expression of epiregulin and amphiregulin on outcome of patients with metastatic colorectal cancer treated with 5-FU/LV 
plus irinotecan or irinotecan plus oxaliplatin as first-line treatment (FIRE 1-trial). Int J Cancer 2016;138:739-746.

65. Jing C, Jin YH, You Z, Qiong Q, Jun Z. Prognostic value of amphiregulin and epiregulin mRNA expression in metastatic colorectal cancer patients. Oncotarget 2016;7:55890-55899.

66. Fernández-Marcelo T, Sánchez-Pernaute A, Pascua I, et al. Clinical relevance of telomere status and telomerase activity in colorectal cancer. PLoS One 2016;11:e0149626.

67. Tariq K, Ghias K. Colorectal cancer carcinogenesis: a review of mechanisms. Cancer Biol Med 2016;13:120-135.

68. Kudryavtseva AV, Lipatova AV, Zaretsky AR, et al. Important molecular genetic markers of colorectal cancer. Oncotarget 2016;7:53959-53983.

69. Arora H, Qureshi R, Rizvi MA, Shrivastava S, Parihar MS. Study of apoptosis-related interactions in colorectal cancer. Tumour Biol 2016;37:14415-14425.

70. Scalise JR, Poças RC, Caneloi TP, et al. DNA damage is a potential marker for TP53 mutation in colorectal carcinogenesis. J Gastrointest Cancer 2016;47:409-416.

71. Narayan S, Sharma R. Molecular mechanism of adenomatous polyposis coli-induced blockade of base excision repair pathway in colorectal carcinogenesis. Life Sci 2015;139:145-152.

72. Kinzler KW, Vogelstein B. Lessons from hereditary colorectal cancer. Cell 1996;87:159-170.

73. Creemers EE, Tijsen AJ, Pinto YM. Circulating microRNAs: novel biomarkers and extracellular communicators in cardiovascular disease? Circ Res 2012;110:483-495.

74. Weissmann-Brenner A, Kushnir M, Lithwick Yanai G, et al. Tumor microRNA-29a expression and the risk of recurrence in stage II colon cancer. Int J Oncol 2012;40:2097-2103.

75. Li Y, Zhang Z. Potential microRNA-mediated oncogenic intercellular communication revealed by pan-cancer analysis. Sci Rep 2014;4:7097.

76. Ma Y, Zhang P, Wang F, et al. miR-150 as a potential biomarker associated with prognosis and therapeutic outcome in colorectal cancer. Gut 2012;61:1447-1453.

77. Liu H, Du L, Wen Z, et al. Up-regulation of miR-182 expression in colorectal cancer tissues and its prognostic value. Int J Colorectal Dis 2013;28:697-703.

78. Akagi Y, Kinugasa T, Adachi Y, Shirouzu K. Prognostic significance of isolated tumor cells in patients with colorectal cancer in recent 10-year studies. Mol Clin Oncol 2013;1:582-592.

79. Tan CR, Zhou L, El-Deiry WS. Circulating tumor cells versus circulating tumor DNA in colorectal cancer: pros and cons. Curr Colorectal Cancer Rep 2016;12:151-161.

80. Siravegna G, Bardelli A. Blood circulating tumor DNA for non-invasive genotyping of colon cancer patients. Mol Oncol 2016;10:475-480.

81. Gingras I, Salgado R, Ignatiadis M. Liquid biopsy: will it be the 'magic tool' for monitoring response of solid tumors to anticancer therapies? Curr Opin Oncol 2015;27:560-567.

82. Dhaliwal A, Vlachostergios PJ, Oikonomou KG, Moshenyat Y. Fecal DNA testing for colorectal cancer screening: Molecular targets and perspectives. World J Gastrointest Oncol 2015;7:178-183.

83. Guinney J, Dienstmann R, Wang X, et al. The consensus molecular subtypes of colorectal cancer. Nat Med 2015;21:1350-1356.

84. Gomez-Roca CA, Delord J, Robert C, et al. Pencorafenib (LGX818), an oral braf inhibitor, in patients (pts) with braf v600e metastatic colorectal cancer $(\mathrm{mcrc})$ : results of dose expansion in an openlabel, phase 1 study. Ann Oncol 2014;25(Suppl 4):iv182-iv183.

85. Kopetz S, Desai J, Chan E, et al. Phase II pilot study of vemurafenib in patients with metastatic BRAF-mutated colorectal cancer. J Clin Oncol 2015;33:4032-4038.

86. Hyman DM, Puzanov I, Subbiah V, et al. Vemurafenib in multiple nonmelanoma cancers with BRAF V600 mutations. $N$ Engl J Med 2015;373:726-736

87. Hong DS, Morris VK, El Osta B, et al. Phase IB Study of vemurafenib in combination with irinotecan and cetuximab in patients with metastatic colorectal cancer with BRAFV600E mutation. Cancer Discov 2016;6:1352-1365.

88. Corcoran RB, André T, Yoshino T, et al. Efficacy and circulating tumor DNA (ctDNA) analysis of the BRAF inhibitor dabrafenib (D), MEK inhibitor trametinib (T), and anti-EGFR antibody panitumumab $(\mathrm{P})$ in patients (pts) with BRAF V600E-mutated (BRAFm) metastatic colorectal cancer (mCRC). Ann Oncol 2016;27:abstract 455O.

89. Tabernero J, Geel RV, Guren TK, et al. Phase 2 results: encorafenib (ENCO) and cetuximab (CETUX) with or without alpelisib (ALP) in patients with advanced BRAF-mutant colorectal cancer (BRAFm CRC). J Clin Oncol 2016;34:abstract 3544. 